Pacific Journal of Mathematic 


\section{REDUCING THE ORDER OF A LAGRANGIAN}

\section{RICHARD ARENS}

Consider a Lagrangian density $L$ defined on $\boldsymbol{R}^{m}$ for a system with configuration space $Q$. Let the order of the highest order derivatives in $L$ be $N$. Then the Euler equations are generally of order $2 N$. We present ways of replacing $L$ by other Lagrangian densities $L^{\prime}$ on $\boldsymbol{R}^{m}$ which are of order 1 and in fact linear in the derivatives. This is done by introducing roughly $n$ times

$$
\left(\begin{array}{r}
m+N-1 \\
N-1
\end{array}\right)+\left(\begin{array}{c}
m+N \\
N
\end{array}\right)-2
$$

additional variables, where $n$ is the dimension of $Q$.

One of these $L^{\prime}$ (denoted by $L^{\wedge}$ ) is such that its Euler equations have a canonical form reducing to that of Hamilton for $N=m=1$.

2. Notation. A Lagrangian density $L$ is a function $F$ of several variables, first the cartesian coordinates

$$
t^{1}, \cdots, t^{m}
$$

in $\boldsymbol{R}^{m}$, and then the coordinates

$$
x^{1}, \cdots, x^{n}
$$

in some manifold $Q$, and also

$$
x_{i}^{2}, \quad x_{i j}^{\lambda}, x_{i j k}^{\lambda}, \cdots
$$

where roman indices range from 1 to $m$ and greek indices from 1 to $n$. Here $i \leqq j, i \leqq j \leqq k$. The greatest number of subscripts on any letter occurring in $L$ is the order of $L . L$ is used to define a functional $\Lambda$ on the class of suitable differentiable maps $f$ from $\boldsymbol{R}^{m}$ to $Q$. In $L=F(t, x, \cdots)$ one replaces each $x^{\lambda}$ by the component $f^{\lambda}=x^{\lambda} \circ f$, and replaces $x_{i}^{\lambda}$ by $\partial f^{\lambda} / \partial t^{i}, x_{i j}^{\lambda}$ by $\partial^{2} f^{\lambda} / \partial t^{i} \partial t^{j}$ ect., and defines $\Lambda(f)$ by

$$
\int_{I} L \circ D f d t^{1} \cdots d t^{m}
$$

The notation $L \circ D f$ is supposed to show that the values of $f$ and its derivatives have been inserted into $L$.

We are going to meet variables with suffixes of their own, and indeed varying from 1 to $m$, so this notation will have to be amended. If $y_{14}$ is some variable occurring in $L$, let $w=y_{14}$, then one 
might also have $w_{2}$, in the sense of (2.1). It would be confusing to write $y_{142}$. We will therefore use parentheses to indicate those variables which are intended to be replaced by derivatives when $\Lambda(f)$ is formed. More precisely, those variables whose description follows (2.1) will be denoted by

$$
\left(x^{2}\right)_{i}, \quad\left(x^{\lambda}\right)_{i j}, \cdots .
$$

When $m=1$, then $\left(x^{\lambda}\right)_{1}$ is the familiar $\dot{x}^{\lambda}\left([1\right.$, p. 178] $)$ and $\left(x^{\lambda}\right)_{11}$ is $\ddot{x}^{\lambda}$.

In the sequel, we will abbreviate an expression like

$$
F\left(t^{1}, \cdots, t^{m}, x^{1}, \cdots, x^{n},\left(x^{1}\right)_{1},\left(x^{1}\right)_{2}, \cdots,\left(x^{n}\right)_{m},\left(x^{1}\right)_{11}, \cdots\right)
$$

by

$$
F\left(t^{i}, x^{2},\left(x^{\lambda}\right)_{\imath},\left(x^{2}\right)_{i j}, \cdots\right)
$$

3. Exploding a Lagrangian density. Let $L=F\left(t^{2}, x^{2},\left(x^{i}\right)_{i}\right.$, $\left.\left(x^{\lambda}\right)_{2 j}, \cdots\right)$ be a Lagrangian density. Then its exploded form is

$$
\begin{aligned}
& L^{*}=F\left(\iota^{i}, x^{\lambda}, x_{\imath}^{\lambda}, x_{i j}^{\lambda}, \cdots\right) \\
& \quad+p_{i}^{i}\left[\left(x^{\lambda}\right)_{\imath}-x_{i}^{\lambda}\right]+p_{k}^{i j}\left[\left(x_{\imath}^{\lambda}\right)_{j}-x_{i j}^{\lambda}\right]+\cdots .
\end{aligned}
$$

Here the

$$
x_{i}^{\lambda}, \quad x_{i j}^{i}, \cdots, p_{\lambda}^{i}, \quad p_{\lambda}^{i j}, \quad p_{k}^{i j k}, \cdots
$$

are new variables. The multiple indices $i j, i j k$ are subject to the restrictions $1 \leqq i \leqq j \leqq m, 1 \leqq i \leqq j \leqq k \leqq m$, etc. We use the summation convention for each pair of (possibly multiple) indices, one high and the other low, summing over all possibilities subject to the restrictions.

Let $L$ have order $N$. Then the expansion in 3.1 is intended to stop after the terms involving $x_{i_{1} i_{2} \cdots i_{N}}^{\lambda}$ have been written down, but it does not hurt the formalism to go beyond this limit.

The new variables will have to be identified as coordinates in some new manifold $\mathscr{B}$. We will take this up later.

It is evident that

$$
\text { the exploded Lagrangian density is of order } 1 .
$$

In the next section we prove the following.

THEOREM 3.4. There is a 1:1 correspondence between the extremals for $L$ and those of $L^{*}$.

Here is the correspondence. Let $f$ be an extremal for $L$, that is, a solution of the relevant Euler equations. These are (cf. [2, p. 
568])

$$
\frac{\partial L}{\partial x^{\lambda}}-\frac{\partial}{\partial t^{i}} \frac{\partial L}{\partial\left(x^{\lambda}\right)_{i}}+\frac{\partial^{2}}{\partial t^{i} \partial t^{j}} \frac{\partial L}{\partial\left(x^{\lambda}\right)_{i j}}+\cdots=0 .
$$

Here $\lambda=1, \cdots, m$ and we are using the same summation convention as before. Thus $1 \leqq i \leqq j \leqq m$, etc.

We now make a new function $D_{L} f$, mapping $\boldsymbol{R}^{m}$ into $\mathscr{B}$ (where the $t^{i}$, the $x^{\lambda}$ and the (3.2) are coordinates). The image $D_{L} f\left(\tau^{1}, \cdots, \tau^{m}\right)$ of $\left(\tau^{1}, \cdots, \tau^{m}\right) \in \boldsymbol{R}^{m}$ under $D_{L} f$ shall be the point where $t^{i}=\tau^{i}$, $x^{\lambda}=f^{\lambda}\left(\tau^{1}, \cdots, \tau^{m}\right)$,

$$
x_{i}^{\lambda}=\frac{\partial f^{\lambda}}{\partial t^{i}}, \quad x_{i j}^{\lambda}=\frac{\partial^{2} f^{\lambda}}{\partial t^{i} \partial t^{j}}, \cdots,
$$

all evaluated at $\left(\tau^{1}, \cdots, \tau^{m}\right)$. Moreover, at $D_{L} f\left(\tau^{1}, \cdots, \tau^{m}\right)$ the $p$ 's shall have the values $p_{\lambda}^{i}=\partial L / \partial\left(x^{\lambda}\right)_{i}, p_{\lambda}^{i j}=\partial L / \partial\left(x^{\lambda}\right)_{i j}, \cdots$. These latter partial derivatives are evaluated at the $t^{i}, x^{\lambda}, x_{i}^{\lambda}$, etc. just specified.

Now (3.4) asserts that $D_{L} f$ is an extremal for $L^{*}$ and that every extremal for $L^{*}$ has this form.

This is more than the following observation: $D_{L} f$ is an extremal for $L^{*}$ if and only if $f$ is an extremal for $L$. In fact, suppose $f$ is not an extremal for $L$, then $D_{L} f$ is not one for $L^{*}$ because

$$
L^{*} \circ D\left(D_{L} f\right)=L \circ D f,
$$

due to the $\left(x^{\lambda}\right)_{i}-x_{i}^{\lambda}$ (etc.) vanishing for $D_{L} f$. Thus if the integral of $L \circ D f$ can be varied, so can the integral of $L^{*} \circ D\left(D_{L} f\right)$. Conversely, if $f$ is an extremal for $L$, then, by looking at the Euler equations for $L^{*}$, given in the next section, one can see that $D_{L} f$ is an extremal. (A similar remark holds for $L^{\wedge}$ to be introduced below.)

In the next section we complete the proof of (3.4) by showing that every extremal for $L^{*}$ has the form $D_{L} f$. (It then follows that $f$ is an extremal for $L$, of course.)

4. Proof of (3.4). We will deal with the case where $L$ has order not greater than 2. Thus we have

$$
\begin{gathered}
L^{*}=F\left(t^{i}, x^{\lambda}, x_{i}^{\lambda}, x_{i j}^{\lambda}\right)+p_{\lambda}^{i}\left[\left(x^{\lambda}\right)_{i}-x_{i}^{\lambda}\right] \\
+p_{\lambda}^{i j}\left[\left(x_{i}^{\lambda}\right)_{j}-x_{i j}^{\lambda}\right] .
\end{gathered}
$$

Since $L^{*}$ is always of the first order, we have for each variable $v$ in $L^{*}$, an Euler equation

$$
\frac{\partial L^{*}}{\partial v}-\frac{\partial}{\partial t^{i}} \frac{\partial L^{*}}{\partial(v)_{i}}=0 .
$$


Consider the case $v=p_{\lambda}^{i j}$. Because there is no $\left(p_{\lambda}^{i j}\right)_{k}$, we get $\left(p_{k}^{i j}\right)$

$$
\left(x_{i}^{\lambda}\right)_{j}-x_{i j}^{\lambda}=0 .
$$

In the same way

$$
\left(x^{\lambda}\right)_{i}-x_{i}^{\lambda}=0 .
$$

Now consider $v=x_{i j}^{\lambda}$. There is no $\left(x_{i j}^{\lambda}\right)_{k}$, but $x_{i j}^{\lambda}$ does occur in the $F$ (as well as in the added terms). Let us denote the partial derivative by

$$
F_{x_{i j}^{2}}^{\lambda} .
$$

(It is the same as first calculating

$$
\frac{\partial L}{\partial\left(x^{\lambda}\right)_{i j}}
$$

and then peeling the parentheses off.) In any case, we have the Euler equations

$$
\begin{array}{cc}
\left(x_{i j}^{\lambda}\right) & F_{x_{i j}^{\lambda}}+p_{\lambda}^{i j}(-1)=0, \\
\left(x_{i}^{\lambda}\right) & F_{x_{i}^{\lambda}}-p_{\lambda}^{i}-\frac{\partial}{\partial t^{j}} p_{\lambda}^{i j}=0, \\
\left(x^{\lambda}\right) & F_{x^{\lambda}}-\frac{\partial}{\partial t^{j}} p_{\lambda}^{j}=0, \quad \lambda=1,2, \cdots, n .
\end{array}
$$

We leave it to the reader to verify that this last set of $n$ equations, in the presence of the preceeding ones, is the set of Euler equations 3.5 for $L$. This completes the proof.

5. Restricting $L^{*}$ to submanifolds. In order to achieve the conclusion of (3.4) it is usually not necessary to use $L^{*}$. The result remains true if $L^{*}$ is restricted to the submanifold defined by the Euler equations of frontier type, to be defined presently. Let us say a set of indices $(\lambda ; i, \cdots, j, k)$ is of interior type if $\left(x^{\lambda}\right)_{i \cdots j k}$ or some $\left(x^{\lambda}\right)_{i \cdots j k \cdots m}$ occurs in $L$. An interior set of indices such as the one just mentioned is of frontier type if no $\left(x^{\lambda}\right)_{i \cdots j k \cdots m}$ occurs in $L$. A variable $x_{i \cdots k}^{\lambda}$ or $p_{\lambda}^{i \cdots k}$ is of interior type or of frontier type, according to the type of its indices. The following is relatively obvious.

THEOREM 5.1. The extremals for $L^{*}$ remain the same if the terms involving the $p_{\lambda}^{i \cdots k}$ not of interior type are omitted from (3.1.)

The Euler equations of frontier type are the Euler equations 
for the variables $x_{i \ldots k}^{\lambda}$ of frontier type. Let us denote the restriction of $L^{*}$ to this "frontier" submanifold by $L^{\wedge}$.

It is easy to see that $L^{\wedge}$ has an expansion like (3.1) where, however, the terms involving the $p_{2}^{i \cdots j k}$ of frontier type replaced by

$$
F_{x_{i \cdots j k}^{\lambda}}\left[\left(x_{i \cdots j}^{\lambda}\right)_{k}-x_{i \cdots j k}^{\lambda}\right] \text {. }
$$

By an extremal for $\hat{L}$ we mean an $f$ defined on $\boldsymbol{R}^{m}$ with values in this frontier submanifold such that the integral of $L^{\wedge}$ is stationary for variations also constrained to the frontier submanifold.

We shall now consider the Hessian matrix

$$
\left(\frac{\partial^{2} F}{\partial x_{i \cdots k}^{\lambda} \partial x_{r \cdots t}^{\mu}}\right)
$$

formed with all the frontier indices $(\lambda ; i, \cdots, k),(\mu ; r, \cdots, t)$. Let us call its determinant $\Delta$.

THEOREM 5.2. Suppose $\Delta$ is not 0 along some extremal for $L^{\wedge}$. Then the functions expressing the $x^{\lambda}$ in terms of the t's give an extremal for $L$.

Proof. Roughly speaking, most of the Euler equations for $L^{*}$ will be given by those for $L^{\wedge}$. $L^{\wedge}$ does not explicitly deliver these following Euler equations for $L^{*}$ (where $x_{i \ldots j k}^{\lambda}$ is a frontier variable) $\left(x_{i \cdots j k}^{\lambda}\right)$

$$
0=F_{x_{i \cdots j k}^{2}}-p_{\lambda}^{i \cdots j k}
$$

and

$\left(p_{\lambda}^{i \cdots j k}\right)$

$$
0=\left(x_{i \cdots j}^{\lambda}\right)_{k}-x_{i \cdots j k}^{\lambda} .
$$

Now the first of these is provided by our extremal for $L^{\wedge}$ because the extremal lies in the frontier submanifold. We now consider an Euler equation for $L^{\wedge}$, namely $\left(x_{r \cdots s t}^{\mu}\right) \quad 0=F_{x_{r}^{\prime} \cdots s t}^{\mu}$

$$
+\sum_{\lambda, i \cdots j k} F_{x_{i \cdots j k}^{\lambda} x_{r \cdots s t}^{\mu}}\left[\left(x_{i \cdots j}^{\lambda}\right)_{k}-x_{i \cdots j k}^{\lambda}\right]-F_{x_{r \cdots s t}^{\mu}}^{\lambda} .
$$

By the non-vanishing of $\Delta$ we conclude that the $x_{\imath \cdots j k}^{\lambda}$ relations for $L^{*}$ hold. Thus we have an extremal for $L$.

As already remarked, an extremal for $L$ always gives one for $L^{\wedge}$.

In classical dynamics $(N=m=1)$ one regularly imposes a condition of non-singularity on the very Hessian which is involved here for $N=m=1$ (cf. [8, p. 339]), of course for other reasons. We give an example showing that this condition cannot be dropped 
from (5.2). Let $Q=R$ and $L=x+x(\dot{x})^{2}$, where we use the classical $\dot{x}$ instead of our present notation $(x)_{1}$. Then $L^{*}=x+x\left(x_{1}\right)^{2}+$ $p\left(\dot{x}-x_{1}\right), \quad L^{\wedge}=x+x\left(x_{1}\right)^{2}+2 x x_{1}\left(\dot{x}-x_{1}\right)=x-x\left(x_{1}\right)^{2}+2 x x_{1} \dot{x} . \quad$ The Euler equations are

$$
\begin{gathered}
1-\left(x_{1}\right)^{2}+2 x_{1} \dot{x}=\frac{d}{d t}\left(2 x x_{1}\right)=2 \dot{x} x_{1}+2 x \dot{x}_{1}, \\
-2 x x_{1}+2 x \dot{x}=0 .
\end{gathered}
$$

Now $x=0$ and $x_{1}=1$ is evidently an extremal for $L^{\wedge}$, but $x=0$ is not an extremal for $L$, whose Euler equation is

$$
1+\dot{x}^{2}=\frac{d}{d t} 2 x \dot{x} .
$$

On the other hand, there are examples from mathematical physics in which the Hessian is singular. Consider the Lagrangian density $[7,111.1]$.

$$
L=\left[(x)_{11}+(x)_{22}\right]^{2}-2 g x .
$$

Here $m=2, n=1, N=2$, and $g$ is supposed to depend only on the $t$ 's. I write down $L^{*}$ :

$$
\begin{aligned}
L^{*}= & \left(x_{11}+x_{22}\right)^{2}-2 g x+p^{1}\left[(x)_{1}-x_{1}\right]+p^{2}\left[(x)_{2}-x_{2}\right] \\
& +p^{11}\left[\left(x_{1}\right)_{1}-x_{11}\right]+p^{12}\left[\left(x_{1}\right)_{2}-x_{12}\right]+p^{22}\left[\left(x_{2}\right)_{2}-x_{22}\right] .
\end{aligned}
$$

To define $L^{\wedge}$ we first drop the $p^{12}$ term and then invoke the relations

$\left(x_{11}\right)$

$\left(x_{22}\right)$

$$
\begin{aligned}
& 2\left(x_{11}+x_{22}\right)-p^{11}=0 \\
& 2\left(x_{11}+x_{22}\right)-p^{22}=0 .
\end{aligned}
$$

Since we cannot solve these for $x_{11}$ and $x_{22}$ the determinant $\Delta$ must be 0 .

6. The Hamiltonian density. $L^{*}$ is the sum two functions: one containing no derivatives, and another which is a homogeneous linear form in derivatives. The same is true for $L^{\wedge}$, and in fact

$$
L^{\wedge}=-H+\Psi
$$

where $\Psi$ is a sum of terms of the form

$$
p_{\lambda}^{i \cdots j k}\left(x_{i \cdots j}^{2}\right)_{k} .
$$

Let us say we have the regular case when the Hessian $\Delta$ (see 5.2) is not 0. Then we can use the Euler equations for the frontier 
variables $x_{i \cdots k}^{\lambda}$ to express (locally, of course) these variables in terms of the frontier $p_{\lambda . . .}^{i}$, and so

we can express $H$ in terms of terms of the interior (including frontier) $p_{\lambda}^{i \cdots k}$ and the interior excluding the frontier variables $x_{i \cdots k}^{\lambda}$.

Understanding, then, that these are taken as coordinates, we can write the Euler equations for $L^{\wedge}$ in the following canonical way:

$$
\left(x_{i \cdots j}^{\lambda}\right) \quad-\frac{\partial H}{\partial x_{i \cdots j}^{\lambda}}-\frac{\partial}{\partial t^{k}} p_{\lambda}^{i \cdots j k}=0,
$$

remembering that one must sum on $k$; and

$\left(p_{\lambda}^{i \cdots j k}\right)$

$$
-\frac{\partial H}{\partial p_{\lambda}^{i \cdots j k}}+\left(x_{i \cdots j}^{\lambda}\right)_{k}=0 .
$$

These latter equations (for example $p_{\lambda}^{i}$ ) means

$$
\frac{\partial x^{\lambda}}{\partial t^{i}}=H_{p_{\lambda}^{i}} .
$$

These equations obviously deserve to be called the canonical equations. ${ }^{1}$

In the classical case $N=m=1$ these are Hamilton's canonical equations, of course. For $m=1$ but $N>1$, the possibility of writing a variational problem in this way was already shown by Whittaker [8, Sec. 110]. The subject was treated in a coordinate free way by Rodrigues [6].

In the non-regular case, this system of canonical equations, i.e., the Euler equations for $L^{\wedge}$, must necessarily contain some strangelooking equations such as the the $p$ ) equation below. After all, in classical mechanics, the Hessian $\Delta$ is required to be nonzero.

We wrote them here under the assumption of the regular case. Problems of mathematical physics may not be regular, for example the one presented in the last section. This does not necessarily exclude a Hamiltonian form. Using the equations $\left(x_{11}, x_{22}\right)$ we obtain

$$
L^{\wedge}=-H+p^{1}(x)_{1}+p^{2}(x)_{2}+p\left[\left(x_{1}\right)_{1}+\left(x_{2}\right)_{2}\right]
$$

where $p=p^{11}\left(=p^{22}\right)$ and

$$
H=(p)^{2} / 4+2 g x+p^{1} x_{1}+p^{2} x_{2} .
$$

Euler's equations for $L^{\wedge}$ are

$$
-2 g=\frac{\partial p^{1}}{\partial t^{1}}+\frac{\partial p^{2}}{\partial t^{2}}
$$

${ }^{1}$ There are $n[(m+N-1 / N-1)]$ of the $(x)$ equations and $n[(m+N / N-1)]$ of the $(p)$ equations. 


$$
\begin{aligned}
& -p^{1}=\frac{\partial p}{\partial t^{1}} \\
& -p^{2}=\frac{\partial p}{\partial t^{2}}
\end{aligned}
$$

$$
-x_{1}+(x)_{1}=0
$$

$$
-x_{2}+(x)_{2}=0
$$

$$
-p / 2+\left(x_{1}\right)_{1}+\left(x_{2}\right)_{2}=0 \text {. }
$$

Now, what has this canonical system to do with (5.3)? We know that an extremal for (5.3) gives a solution to the canonical system, since it is based on $L^{\wedge}$. Elimination of the $p$ 's, however, shows that our system implies

$$
g=\left[\left(\frac{\partial}{\partial t^{1}}\right)^{2}+\left(\frac{\partial}{\partial t^{2}}\right)^{2}\right]^{2} x
$$

This is the Euler equation for (5.3). Thus the canonical system does reduce (6.1) to a system of six first order equations.

7. Setting up a Cauchy problem. We show that having a system of partial differential equation in canonical form often leads to a Cauchy problem. We take only the case $N=1$, so that the original Euler equations form a system of $n$ second order equations.

THEOREM 7.1. Suppose that the Hessian

$$
\left(\frac{\partial^{2} H}{\partial p_{\lambda}^{i} \partial p_{\mu}^{j}}\right) i, j=1,2, \cdots m-1 ; \lambda, \mu=1, \cdots, n
$$

is not 0 at some point in $t^{i}, p_{\lambda}^{i}, x^{\lambda}$ space. Then there is a system of differential equations

$$
\left\{\begin{array}{l}
\frac{\partial}{\partial t^{m}} p_{\lambda}^{i}=A_{\lambda}^{i} \\
\frac{\partial}{\partial t^{m}} x^{\lambda}=B^{\lambda}
\end{array}\right.
$$

where $A_{\lambda}^{i}$ and $B^{\lambda}$ are first degree polynomials in the $\partial / \partial t^{k}$ of $p_{\mu}^{j}$ and $x^{\mu}$ with $k<m$ such that any solution of (7.2) with initial conditions including

$$
\frac{\partial x^{\lambda}}{\partial t^{k}}=H_{p_{\lambda}^{k}} \quad(k=1, \cdots, m-1)
$$

gives a solution of the canonical equations. 
In the proof we will consider only the case $n=1$ and $m=2$, because it is very easy to generalize from this case. We will also write $s$ for $t^{1}, t$ for $t^{2}, p$ for $p^{1}$ and $q$ for $p^{2}$.

The canonical equations to be solved are

$$
\frac{\partial p}{\partial s}+\frac{\partial q}{\partial t}=-H_{x}, \quad \frac{\partial x}{\partial s}=H_{p}, \quad \frac{\partial x}{\partial t}=H_{q} .
$$

Two of the three equations in the system (7.2) shall be

$$
\frac{\partial q}{\partial t}=-H_{X}-\frac{\partial p}{\partial s}, \frac{\partial x}{\partial t}=H_{p} .
$$

We need a formula for $\partial p / \partial t$. We can obtain one by equating $\partial^{2} x / \partial s \partial t$ and $\partial^{2} x / \partial t \partial s$. More explicitly, we set

$$
\frac{\partial}{\partial t} H_{p}=\frac{\partial}{\partial s} H_{q} \text {. }
$$

This leads to

$$
H_{p p} \frac{\partial p}{\partial t}=H_{q s}-H_{p t}+H_{q x} H_{p}-H_{p x} H_{q}+H_{p q} H_{p}+2 H_{p q} \frac{\partial p}{\partial s}+H_{q q} \frac{\partial q}{\partial s} .
$$

Dividing by $H_{p p}$, which is not 0 by hypothesis, gives an equation which we nominate to be the third member of (7.2).

We now suppose we have a solution of (7.2) with $x(s, 0)=f(s)$, $p(s, 0)=g(s), q(s, 0)=h(s)$, and $H_{p}(s, 0, f(s), q(s), h(s))=f^{\prime}(s)$. We want to know if

$$
Z(s, t)=x_{1}(s, t)-H_{p}(s, t, x(s, t), p(s, t), q(s, t))
$$

will continue to be 0 for $t$ near 0 . Calculation shows that

$$
\frac{\partial Z}{\partial t}=H_{q x} Z \text {. }
$$

So $Z(s, 0)=0$ implies $Z(s, t)=0$, as desired.

For greater $m$ and $n$ we discover the extra equations for (7.2) by equating

$$
\frac{\partial}{\partial t^{m}} H_{p_{\lambda}^{i}} \text { and } \frac{\partial}{\partial t^{i}} H_{p_{\lambda}^{m}}
$$

8. The manifolds involved. Let $M$ and $Q$ be differentiable manifolds, with $\operatorname{dim} M=m$ and $\operatorname{dim} Q=n$. Let $N \geqq 1$ be an integer. Let $f, g$ be maps of $M$ into $Q$ with continuous derivatives of order $N$ at least. Let $P$ be a point of $M$. Say that $f \equiv g$ at $P$ if $f$ and $g$ have the same value, and the same partial derivatives of 
all orders up to and including the $N$ th, at $P$. Denote the collection of equivalence classes by $J^{(N)}(M, Q)_{P}$. Denote the union for all $P$ by $J^{(N)}(M, Q)$, following the notation of [4]. Coordinates are defined as follows. Let $t^{1}, \cdots, t^{m}$ and $x^{1}, \cdots, x^{n}$ be coordinate systems in $M$ and $Q$ respectively and suppose the $t^{i}$ are defined at $P$ and that the $x^{\lambda}$ are defined at $f(P)$. Let $C$ be the element of $J^{(N)}(M, Q)_{P}$ containing $f$. Then ${ }^{2)} \bar{t}^{i}(C)$ shall be $t^{i}(P)$ and $x^{\lambda}(C)$ shall be $\bar{x}^{\lambda}(f(P))$. For $1 \leqq k \leqq N$

$$
\left(x^{\lambda}\right)_{i_{1} \cdots i_{k}}(C)=\left.\frac{\partial^{k}}{\partial t^{i_{1} \cdots \partial t^{i_{k}}}}\left(x^{\lambda} \circ f\right)\right|_{P} .
$$

Let $f: M \rightarrow Q$. Then we define

$$
D f: M \longrightarrow J^{(N)}(M, Q)
$$

by making $(D f)(P)=C$ where $C$ is the class at $P$ to which $f$ belongs. When $M=\boldsymbol{R}^{m}$ we can define a Lagrangian density $L$ as a real-valued function on $J^{(N)}\left(\boldsymbol{R}^{m}, Q\right)$. Therefore $L^{*}$ (3.1) must also be defined on some $J^{(N)}\left(\boldsymbol{R}^{m}, Q\right)$ with some new choice of $N, Q$ and possibly $m$.

In fact, $L^{*}$ is defined on $J^{(1)}\left(\boldsymbol{R}^{m}, K\right)$ where $K$ is $J^{(1)}(J, R)$ and $J$ is $J^{(N)}\left(\boldsymbol{R}^{m}, Q\right)$ where $N$ here is the order of the Lagrangian $L$ under consideration. To bring this out, we shall repeat here the Definition 3.1 using the meticulous notation

$$
\begin{aligned}
& \left.\left.L^{*}=F\left(\overline{\overline{t^{i}}}, \overline{\overline{x^{\lambda}}}, \overline{\overline{\left(x^{\lambda}\right.}}\right)_{i}, \cdots\right)+\overline{p_{\lambda}^{i}}\left[\overline{\overline{\left(x^{\lambda}\right.}}\right)_{i}-\left(\overline{\overline{\left.x^{\lambda}\right)_{i}}}\right)\right]
\end{aligned}
$$

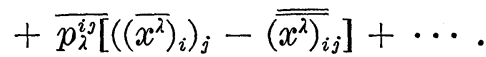

The $t^{i}$ and $x^{\lambda}$ here arose as coordinates in $M \times Q$ so the three bars make sense for an $L^{*}$ defined on a bundle three levels above $M \times Q$.

Here is a table, to be read from the bottom up, indicating the bundles and the coordinates.

$$
\begin{array}{ll}
J^{(1)}\left(\boldsymbol{R}^{m}, K\right) & \overline{\overline{t^{i}}} \overline{\overline{x^{\lambda}}} \overline{\overline{\left(x^{\lambda}\right)_{i}}} \overline{\overline{\left(x^{\lambda}\right)_{i}}} \cdots \overline{p_{\lambda}^{i}} \overline{p_{\lambda}^{i j}} \cdots \\
K=J^{(1)}(J, \boldsymbol{R}) & \overline{\overline{t^{i}}} \overline{\overline{x^{\lambda}}} \overline{\left(x^{\lambda}\right)_{i}} \overline{\left(x^{\lambda}\right)_{i j}} \cdots p_{\lambda}^{i} p_{\lambda}^{i j} \cdots \\
J=J^{(N)}\left(\boldsymbol{R}^{m}, Q\right) & \overline{t^{i}} \overline{x^{\lambda}}\left(x^{\lambda}\right)_{i}\left(x^{\lambda}\right)_{i j} \cdots \\
\boldsymbol{R} \times \boldsymbol{Q} & t^{i} x^{\lambda} .
\end{array}
$$

We now come to the definition of the $p_{\lambda}^{i \cdots j}$. All that the proof requires is that the variables in the second row of the table be in-

${ }^{2}$ These bars will be omitted after they have served their purpose in the exposition. Instead of the bars we could have written $t^{1} \circ \pi$ where $\pi$ is the projection map onto $M \times Q$, and $x^{\lambda_{0}} \pi$. If one wants to be meticulous, the $t^{i}$ and $x^{\lambda}$ in (2.3) have to have these bars over them. 
dependent.

Let $C$ be a point of $K$. Now $C$ is a pair $(A,[f])$ where $A$ is a point of $J$ and $[f]$ is an equivalence class of maps of which one, $f: J \circ \rightarrow \boldsymbol{R}$ shall be selected (the small $\circ$ indicates that $f$ is defined only on an open subset of $J)$. ( $A$ itself is a pair $(B,[g])$ where $g: \boldsymbol{R}^{m} \circ \rightarrow Q, B \in \boldsymbol{R}^{m}$.)

The most useful definition of $p_{\lambda}^{i \cdots j}(C)$ is to let it be some constant times

$$
\frac{\partial f}{\partial\left(x^{2}\right)_{i \cdots j}}(A) \text {. }
$$

These constants can be chosen so as to make 8.2 independent of the coordinates $x$ used.

\section{REFERENCES}

1. Richard Arens, Differential-geometric elements of analytic dynamics, J. Math. Anal. Appl., 9 (1964), 165-202.

2. A. R. Forsyth, Calculus of Variations, Cambridge University Press, 1927.

3. I. M. Gelfand and S. V. Fomin, Calculus of Variations, Prentice-Hall Inc., Englewood Cliffs, 1963.

4. M. Golubitzsky and V. Guillemin, Stable mappings and their singularities, G.T.M. 14, Springer, 1973.

5. W. F. Osgood, Mechanics, Macmillan, New York, 1937.

6. P. R. Rodrigues, Sur les systèmes mécaniques generalizés, C. R. Acad. Sci. Paris 282, Der. A. (1976), 1307-1309.

7. I. S. Sokolnikoff, Mathematical Theory of Elasticity, McGraw-Hill Book Company, Inc., 1956.

8. E. T. Whittaker, Analytical Dynamics of Particles and Rigid Bodies, Cambridg University Press, 1937.

Received April 24, 1978. This research was supported by NSF grant MCS 76-05857.

UNIVERSITY OF CALIFORNiA

Los Angeles, CA 90024 



\section{PACIFIC JOURNAL OF MATHEMATICS}

\section{EDITORS}

DONALD BABBITT (Managing Editor)

University of Galifornia

Los Angeles, California 90024

HUgo RossI

University of Utah

Salt Lake City, UT 84112

C. C. MOORE AND ANDREW OGG

University of California

Berkeley, CA 94720

\section{J. DUGUNDJI}

Department of Mathematics University of Southern California Los Angeles, California 90007

R. Finn and J. Milgram

Stanford University

Stanford, California 94305

\section{ASSOCIATE EDITORS}
R. ARENS
E. F. BECKENBACH
B. H. NeUManN
F. WOLF
K. YosHIDA

\section{SUPPORTING INSTITUTIONS}

UNIVERSITY OF ARIZONA

UNIVERSITY OF BRITISH COLUMBIA

CALIFORNIA INSTITUTE OF TECHNOLOGY

UNIVERSITY OF CALIFORNIA

MONTANA STATE UNIVERSITY

UNIVERSITY OF NEVADA, RENO

NEW MEXICO STATE UNIVERSITY

OREGON STATE UNIVERSITY
UNIVERSITY OF OREGON UNIVERSITY OF SOUTHERN CALIFONIA STANFORD UNIVERSITY UNIVERSITY OF HAWAII UNIVERSITY OF TOKYO UNIVERSITY OF UTAH WASHINGTON STATE UNIVERSITY UNIVERSITY OF WASHINGTON 


\section{Pacific Journal of Mathematics}

\section{Vol. 93, No. $1 \quad$ March, 1981}

Richard Arens, Reducing the order of a Lagrangian $\ldots \ldots \ldots \ldots \ldots \ldots \ldots$

Richard Arens, Manifestly dynamic forms in the Cartan-Hamilton treatment

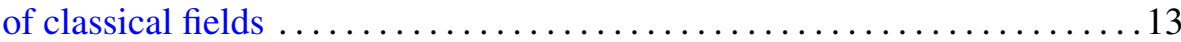

Jimmy T. Arnold, Power series rings over discrete valuation rings $\ldots \ldots \ldots 31$

Charles A. Asmuth and Joe Repka, Supercuspidal components of the

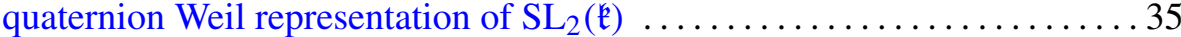

Luis A. Caffarelli and Avner Friedman, Sequential testing of several simple hypotheses for a diffusion process and the corresponding free boundary problem ................................. 49

William B. Jacob, Fans, real valuations, and hereditarily-Pythagorean fields .............................................. 95

W. J. Kim, Asymptotic properties of nonoscillatory solutions of higher order

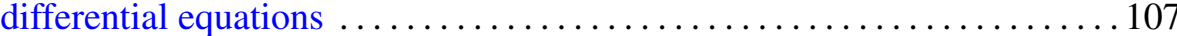

Wayne Steven Lewis, Embeddings of the pseudo-arc in $E^{2} \ldots \ldots \ldots \ldots \ldots 115$

Daniel Alan Marcus, Closed factors of normal Z-semimodules . ......... 121

Mitsuru Nakai and Leo Sario, Harmonic functionals on open Riemann surfaces ............................................. 147

John Currie Quigg, Jr., On the irreducibility of an induced

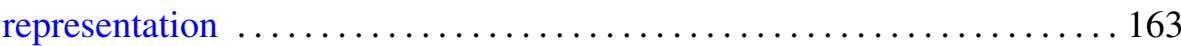

John Henry Reinoehl, Lie algebras and Hopf algebras 181

Joe Repka, Base change for tempered irreducible representations of $\mathrm{GL}(n, \mathbf{R})$

Peter John Rowley, Solubility of finite groups admitting a fixed-point-free automorphism of order $r s t$. I . . . . . . . . . . . . . . . . . . 201

Alan C. Woods, The asymmetric product of three homogeneous linear forms 